\title{
Local Volunteerism and Resilience Following Large-Scale Disaster: Outcomes for Health Support Team Volunteers in Haiti
}

\author{
Jessica A. Carlile • Kira Mauseth • Noël E. Clark • \\ Jennifer L. Cruz $\cdot$ John W. Thoburn
}

Published online: 16 September 2014

(c) The Author(s) 2014. This article is published with open access at Springerlink.com

\begin{abstract}
In-depth interviews with local Haitian volunteers trained in a psychological disaster recovery program called Health Support Team (HST) provide insight into the psychosocial outcomes resulting from their engagement with the program. Qualitative interviews were conducted with four male Haitian participants who had survived the January 2010 Haiti earthquake and had worked as HST volunteers for at least 6 months. Interviews were analyzed using narrative inquiry analysis, which allows individuals to discover and disclose a deeper meaning in their experience and enables the researchers to access more detailed data. Previous research supports the claim that volunteerism provides many important psychological benefits, and the results of the present study suggest that among survivors of large-scale disasters, volunteerism is beneficial as a means of increasing psychological resilience and facilitating personal recovery. Results and themes of our analysis included a reported increase in both hope and purpose for the respondents. Findings suggest that volunteerism on the part of members of the surviving community following large-scale disaster increases resilience among the volunteers and further contributes to their recovery.
\end{abstract}

Keywords Haiti earthquake - Large-scale disaster $\cdot$ Post-disaster resilience $\cdot$ Recovery process $\cdot$ Volunteerism

J. A. Carlile $(\bowtie) \cdot$ N. E. Clark · J. W. Thoburn Department of Clinical Psychology, Seattle Pacific University, Seattle, WA 98119, USA

e-mail: carlilej@spu.edu

K. Mauseth · J. L. Cruz

Department of Psychology, Seattle University, Seattle, WA 98122, USA

\section{Introduction}

In the context of a large-scale disaster, it is essential to immediately identify resources, interventions, and sustainable practices that will assist the surviving population. In Haiti in 2010, following the devastating January earthquake that killed nearly 300,000 people, there was clear need for resources, interventions, and sustainable practices in which Haitians could engage to contribute to their own long-term recovery. From the overwhelming, often disorganized, and typically short-term influx of foreign aid to Haiti throughout 2010, the unsustainable and finite nature of resources quickly became apparent. Although money was spent, medicine was distributed, and people were assisted, few Haitians were able to become an active part of their recovery process. With some exceptions, many foreigners arrived and departed within months, leaving few accessible tools and resources for Haitians to incorporate into their existing infrastructure or cultural practices.

The Health Support Team (HST) was created as a psychosocial program intended to facilitate Haitian recovery through a train-the-trainer model in which local volunteers would be trained to provide psychological support to friends and family within their own community. The purpose of psychological support in this context is twofold: to assist survivors in their recovery, and to establish sustainable, long-term assistance through the training and empowerment of local volunteers. Psychological support through this program does not include the provision of psychotherapy, but provides crisis intervention with the goal of helping survivors choose effective coping strategies when navigating the aftermath of a disaster. The psychological support offered by local volunteers builds on the strengths of the community and is low cost and sustainable, building on the primary intervention of active listening. 
This community-based support utilizes coping strategies that ultimately strengthen the communities within which they are employed (Jacobs 2007).

There are four primary models of psychological support utilized by the disaster psychology community in providing aid for disaster survivors: the American Red Cross (ARC) model (ARC 2009), the Psychological Triage and Rapid Treatment (PsySTART) model (Schreiber 2010), the psychological first aid (PFA) model (Ruzek et al. 2007), and the critical incident stress management (CISM) model (Mitchell and Everly 2001). While all of these models may be highly useful in disaster settings, each displays some weakness with regard to long-term sustainable practices. The need for a sustainable program with a knowledge base and usability accessible to survivors beyond the acute phase of disaster drove the development of the HST curriculum (Shelly 2011). Haitian HST volunteers were intentionally selected based on informal community connections akin to the snowball technique-a method by which participants recruit future participants from among their acquaintances. The HST program is based on a locally driven and sustainable recovery model that requires selfselection and self-motivation on the part of the volunteers. Therefore, it was essential that the recruitment of volunteers followed a natural and organic process.

The Haitian HST volunteers were trained in basic aspects of disaster mental health support aid (for example, relaxation strategies, cognitive techniques, and psychoeducation skills) that have been demonstrated to facilitate resilience in recovering populations. Content provided to Haitian HST volunteers included information on basic disaster mental health symptoms and evidence-based recovery practices. Specifically, the four modules of the training include information on symptom management, suicide, grief and loss, cognitive coping strategies, and active listening. Haitian HST volunteers were asked to use a four-step process in working with members of their community: (1) listen and learn, (2) offer support, (3) provide tools, and (4) emphasize hope. Volunteers were also trained in how to recognize their own limits, how and when to refer someone for professional help, and how to manage their own compassion fatigue [for more information on the content of the HST curriculum, see Thoburn et al. (2014)]. Following their training, volunteers met individually with members of their community who were experiencing distress. The range of the volunteers' involvement varied greatly and was largely dependent on survivors' desire for support, with Haitian HST volunteers meeting with some survivors only three or four times and in some cases upwards of twenty times.

In 2011, a study was undertaken to evaluate the effectiveness of the HST program. Both Haitian HST volunteers and the survivors supported by those volunteers were interviewed. An initial analysis of the results from interviews obtained from the survivor community members showed positive support for the interventions offered by the Haitian HST volunteers [see Thoburn et al. (2014)]. Each of the survivors who were helped by a Haitian HST volunteer stated that the single most positive benefit they experienced was the impact of another person taking the time to listen to them and hear their story. Survivors also reported that they greatly benefited from the relaxation and coping strategies that were taught.

Although it is clear that psychosocial support from community members is helpful in the development of psychological resilience following large-scale disaster for those who are supported ( $\mathrm{Li}$ and Ferraro 2005; Tang et al. 2010), less is known about the effect on the person doing the supporting-specifically, the extent to which volunteering to help others heal psychologically is beneficial to the resilience of the volunteer. The purpose of the current study was to examine the psychosocial effects of volunteering through the HST program on the volunteers themselves. By examining the HST program through a series of studies, we hope to demonstrate the benefits of the program not only for the survivors, but also for the local volunteers and the greater community. Here we provide initial evidence of how the process of being part of a communitybased disaster mental health program as a trained local volunteer facilitates recovery and resilience within the volunteer.

\subsection{Psychological Benefits of Volunteerism Following Large-Scale Disaster}

Research demonstrates a strong positive correlation between volunteering and psychological well-being (Brown et al. 2012). While volunteers may act out of motivation to perform an act of generosity, they are likely to also indirectly benefit from their experience. Both receiving and providing help may have a significant impact on mental health, but the act of serving others provides the greater psychological benefit (Glister 2012; Hildago et al. 2013). Volunteering is known to enhance psychological well-being through general social interaction (Li and Ferraro 2005; Tang et al. 2010). In the context of large-scale disaster recovery, and particularly in the immediate aftermath, social interaction may be limited and focused on survival-oriented tasks as opposed to psychological recovery.

In an environment with limited resources, connecting to others is an essential element of restoring mental health (Charney 2004; Gelkopf et al. 2008); volunteering can lead to an increased sense of belonging, which may be instrumental in activating personal resilience (King and MacGregor 2000). Volunteering is an emotionally significant activity that strengthens emotional support and promotes 
increased social interaction (Tang et al. 2010). Providing emotional support, especially to disaster victims, allows volunteers to identify with others who are experiencing similar hardships. Connecting with peers further enables volunteers to recognize or to learn from the coping skills utilized by those they are supporting. In this reciprocal manner, providing support for others has the potential to become immeasurably helpful for the volunteer. Volunteering also contributes to an awareness of "social resources" that may be useful in the coping process postdisaster (Brown et al. 2012). In addition to the personal emotional benefits, volunteering can buffer against the negative effects of stressful events (Li and Ferraro 2005). In the context of large-scale disaster recovery, stress is normative. The dual effect of having a buffer from that stress in the form of social interactions, and finding purpose in those interactions as a volunteer may be significant in contributing to resilience.

Most current disaster response interventions are designed to facilitate an increase in resilience. While there are numerous methods for increasing resilience, few have focused on a measurable, systematic way of increasing two known elements of resilience: purpose and connection to others (King and MacGregor 2000). Volunteerism through the HST program may contribute both to purpose and to connection by not only providing the volunteer a task to do, but also by connecting the volunteer to a specific group of people within their own community who will benefit from their service. After receiving multiple trainings and working directly with their peers for several months, one Haitian HST volunteer reported on a Facebook message board designed for HST members: "I am so happy to have this training. It gives me something to do every day. I don't have a job, but now I can do this. I get to help my fellow Haitians and help them get better."

Following natural disasters, those who feel that they are contributing to the well-being of others may experience less stress in comparison with those who do not engage in volunteer work. As psychological well-being and social inclusion are often associated with improved mental health, volunteering is also linked to alleviating depressive symptoms (Glister 2012), and volunteering has been recommended as a long-term intervention to decrease symptoms of depression (Poulin 2014). Recent research continues to demonstrate that individuals experiencing symptoms of depression who volunteer may improve their overall psychological functioning, helping to end long-term depressive episodes (Hildago et al. 2013). After a largescale disaster, volunteering within an affected community may be one of few available opportunities that survivors have to improve their mental health. Li and Ferraro (2005) found that volunteering could successfully be used as an alternative treatment for those who are clinically depressed, which presents implications for disaster mental health work in international locations where psychotropic medication is neither available nor culturally accepted. The concept of equipping disaster survivors with skills to volunteer in recovery efforts within their own communities creates a practical model for contributing to sustainable, community-wide recovery and resilience. It is from this conceptualization, as well as other evidence-based disaster recovery programs, that the HST training was developed and conducted.

\subsection{The Health Support Team (HST) Volunteer Training Process}

The first HST training after the Haiti earthquake was held in May 2010. Eighteen Haitian volunteers and a translator were recruited through organic snowballing techniques to attend the first training in Port-au-Prince. The translator also reached out to his social network, including several university students who had been in the process of pursuing degrees in the social sciences before the earthquake. As a result of this networking, many members of the first group of volunteers were undergraduate students from academic disciplines-including anthropology, sociology, and psychology - who were unable to attend university classes since much of their university had been destroyed in the earthquake. The group was motivated, intrinsically oriented around helping others, and intellectually familiar with the social sciences. These students contacted their friends and classmates, resulting in 18 local individuals at the initial training. The backgrounds of other local volunteers were diverse. On one occasion, for example, the translator was making several copies of the training materials at a local shop. An employee of the shop (22year-old man) was reading the training materials as the copies were being completed. He inquired about the training and was invited to attend. Due to the organic and non-systematic nature of the recruitment process, Haitian HST volunteers were essentially self-selected.

Subsequent trainings were arranged in much the same way, and included members of various social and religious groups who had friends who had attended a previous training and passed along information about the experience and what they had learned. A total of four volunteer trainings in the Port-au-Prince area were conducted free of charge by our team of psychologists from the United States. Each training was 4-6 h in duration with an average of 40 local individuals participating, resulting in approximately 150 Haitian HST volunteers that were trained by the psychologists. While many individuals utilized the information solely for their own personal care, those local Haitians who had attended at least three trainings and who had been actively working with the HST model in their 
communities were invited to become "trained trainers" of the material so that they could begin teaching new groups of additional volunteers. Through the trainings led by the Haitian HST trained trainers in the 2 years following the 2010 earthquake, approximately 350 more volunteers have been trained in the HST curriculum. Due to the rapid growth and apparent utility of the HST program, research was needed to empirically support the effectiveness of the program.

\section{Method}

Narrative inquiry qualitative methodology was utilized to assess the benefits and limitations of the HST curriculum that were experienced by the local Haitian HST volunteers.

\subsection{Participants in the Study}

Participants in this study were recruited from Haitian nationals who had been volunteering with the HST program for at least 6 months. Participants were working within the tent cities extant within the borders of Haiti's capital city, Port-au-Prince. They had been trained as HST volunteers within the previous 2 years. To participate in this study individuals had to meet five criteria: they had to be (1) Haitian nationals; (2) 18 years or older; (3) survivors of the 2010 earthquake; (4) trained HST volunteers; and (5) HST volunteer workers for at least 6 months. Including those individuals trained by the local Haitian HST trained trainers, we estimate that approximately 150 Haitian HST volunteers would meet criteria to participate in our study; however, due to the barriers of recruitment for a study in a post-disaster environment (for example, frequent location changes and lack of phones or permanent addresses), we obtained interviews from 10 HST volunteers. Yet, due to difficulty with transcription, only the complete data from four volunteers were utilized for this study. These four participants were single males, ranging in age from 28 to 37 years, who lived in Port-au-Prince. Three of the four were university students at the time of the earthquake. All participants spoke French-Creole.

In the qualitative literature, no current consensus exists regarding the recommended sample size for narrative inquiry methodology (Richie et al. 2003). Instead, the sample size must reflect the goals and purpose of the individual study; sufficient data are necessary to draw out universal concepts, but the quality of the data is the ultimate measure of its value over the size of the sample (Richie et al. 2003). Creswell et al. (2007) and Lieblich et al. (1998) suggested that it is most important to attain data saturation, which is achieved when there is redundancy of information in interviews, regardless of the sample size. Although we recognize the limitations in deriving complex conclusions from our limited sample size, the commonalities present in the data give credence to the conclusions that have been drawn and warrant attention.

\subsection{Procedure for Interviews}

The procedure for the data collection included interviewing each of the Haitian HST volunteers participating in the study at a location convenient to them in Port-au-Prince and environs. Interviews were conducted by a research team member from the United States and took place in October 2011, as part of a larger research project on the effectiveness of the HST program in general. A translator was used with each participant. The interview process began with the researcher explaining the informed consent and ensuring the full understanding of the participant. Creswell et al. (2007) recommended that narrative interviews utilize several open-ended, broad, topic-specific questions, limiting the use of structure during the interview. Therefore, the interviews were organized and conducted with non-directive, chronologically oriented questions pertaining to the HST and life pre- and postearthquake. Open-ended questions included, "What was your life like before the earthquake?" and "How were you affected by your experience working as an HST volunteer?" An important component of the narrative inquiry process is asking questions that allow the participant to guide his or her responses in a way that is meaningful to him or her (Reissman 1993). Participants' spontaneous answers to the scripted questions were followed by clarifying questions and reflections to draw out greater depth in response. The four interviews were between 40 and $60 \mathrm{~min}$ in duration for each participant.

\subsection{Procedure for Analysis}

Qualitative research provides an exploratory and subjective approach to research (Haverkamp and Young 2007) that is appropriate for unique small samples and is not limited in the study of individual experiences (Reissman 1993; Ponterotto 2005). Qualitative approaches are particularly useful for identifying and conceptualizing the meanings that human beings attribute to events they experience. This method provides information about diverse cultural themes and practices in the lives of unique individuals (Gilgun 2009), which is relevant in assessing the effectiveness of our international post-disaster intervention. An evidence-based and common method of qualitative analysis is narrative inquiry, which allows individuals to discover and disclose a deeper meaning in their experience (Ponterotto 2005). The chronological nature of narrative inquiry creates a linear 
framework that provides researchers with the ability to conduct detailed and coherent analysis that may serve to validate or invalidate preexisting theory. The narrative process further enables the researcher to access detailed data that cannot be acquired through the use of measures, questionnaires, and quantitative research (Creswell et al. 2007; Gilgun 2009). Narrative inquiry permitted each of the Haitian HST volunteers participating in the study to share his experience of post-disaster life in Haiti and his experience with the HST in chronological narrative form; then, through methodical analysis the data from the available four interviews could be extrapolated and reorganized in order to generalize and better understand the effectiveness of the HST and the experience of volunteers pre- and post-disaster.

In accordance with narrative inquiry analysis procedures, each of the available four participants' interviews was listened to and transcribed, and then checked in its entirety to verify accuracy of the transcription (Ponterotto 2005). Following transcription, researchers read the interviews multiple times in order to gain familiarity with the content. Initial impressions regarding the emotional content and overall narrative thread were noted. Researchers then broke interviews into fragments that were grouped into themes, categories, and concepts. Specific passages of the interviews were identified that support the broader concepts. This method of narrative analysis combined the holistic elements of systematic content analysis with detailed procedural analysis. Data saturation was reached after each interview was evaluated for content, categorical, and detail elements, and it was determined that similar themes, categories, and concepts were present across all of the analyzed interviews. Through this approach a detailed understanding of the experience of the four Haitian HST volunteers was reached. Overall, 46 themes, 9 categories, and 3 concepts were identified through the data (Table 1).

\section{Results}

The following summarized results are based on the data from the available four Haitian HST volunteer interviews. To facilitate readability of our study's results, the grammar of participants' quotations was carefully amended while retaining the original content.

To provide a context for the evaluation of HST volunteering, the interview protocol offered an opportunity to contextualize HST by inviting respondents to describe the pre- and post-disaster living conditions in Haiti. We also anticipated that a better understanding of the living environment would provide researchers with useful information for further development of the HST curriculum. Thus, in the interviews, participants retrospectively discussed their lives in Haiti before and immediately after the 2010 earthquake, in addition to sharing their experience with the HST curriculum. Participants consistently reported that life circumstances had been difficult in general prior to the earthquake; they reported financial hardships, but also a sense of purpose in working toward better futures through education and political involvement.

After the earthquake, life in Haiti was not only difficult, but also bleak and marred by tragedy. Participants spoke of the harsh conditions that made them feel as if there were no life left to live in Haiti. One participant stated, "If I ever came back to Port-au-Prince, there would be no addresses, no life-no practical life here." All four participants spoke of the lack of resources, and all experienced the loss of jobs, dislocation, and a loss of a sense of purpose. One participant described the conditions of living immediately following the earthquake as inhumane, stating "in this situation some people were living as if they were animals." Even though international aid agencies provided some

Table 1 Results of the interview analysis of the four interviews with Haitian HST volunteers

\begin{tabular}{lc}
\hline Themes & Categories \\
\hline $\begin{array}{l}\text { Financial struggles, family concerns, school, normal living, routine } \\
\text { Desire for change, discontentment, generally happy }\end{array}$ & $\begin{array}{l}\text { Conditions } \\
\text { Feelings }\end{array}$ \\
$\begin{array}{l}\text { Loss, lack of resources, exposure to death and danger, medical problems, living like animals, joblessness, } \\
\text { poor state of country }\end{array}$ & Conditions \\
$\begin{array}{l}\text { Fear, despair, hopelessness, loss of purpose, stress, helplessness, worry for future, mental health } \\
\text { problems, suffering }\end{array}$ & Feelings \\
$\begin{array}{l}\text { International relief organizations, church groups, dependence on family, limited resources } \\
\text { Desire to help others, immediacy of need, mutual support }\end{array}$ & $\begin{array}{c}\text { Aid } \\
\text { Potivation to } \\
\text { volunteer }\end{array}$ \\
$\begin{array}{c}\text { Active listening, ability to comfort, learned self-care, learned to help others help themselves, able to } \\
\text { manage persistent thoughts }\end{array}$ & $\begin{array}{c}\text { Skill acquisition } \\
\text { Exposure to suffering, fear, lack of resources, translation problems }\end{array}$ \\
$\begin{array}{c}\text { Acquisition of intangible resource, increased social support, increased hope, increased sense of purpose, } \\
\text { provides task to do, changes life situation }\end{array}$ & $\begin{array}{c}\text { Outcomes } \\
\text { HST }\end{array}$ \\
\hline
\end{tabular}


basic resources, women and children were prioritized, which left little support available for many Haitians. Often fighting occurred among Haitians over the limited resources. One participant initially moved out of the city to get away from the violence.

All four participants emphasized universal fear and hopelessness following the earthquake; however, initial coping varied among the participants with two individuals experiencing more severe symptoms of posttraumatic stress. One participant was so affected by the traumatic experience of loss within Port-au-Prince that he chose to relocate outside of the city. Inside the city he reported experiencing difficulty preventing thoughts of the disaster, stating that "all the time I would be feeling scared; I would be smelling dead bodies, and I became hypervigilant because any little noise would make me scared." He reported having nightmares for a year following the earthquake and would often wake up and run out of his house, feeling as though he were reexperiencing the moments when the earthquake happened.

Through working with the HST, participants reported gaining a renewed sense of purpose. Three of the four participants described strong motivations to volunteer and to "help other people like me." Participants had experienced traumatic loss during the earthquake and could therefore identify with the ongoing needs of others in their communities. Participants mostly cited the desire to offer resources to others as a primary reason to volunteer. One participant indicated a secondary motivation of personal gain through volunteering-if he was able to offer something to others, he believed people might see that he was trying to help and share their resources with him as well.

In their assessment of the HST program, the participants reported benefiting from the skills they acquired through the training, particularly active listening and relaxation techniques. Participants found the trainings helpful for their own well-being, as well as for their increased ability to help other survivors. One participant reported a better ability to cope with his own mental health and stated, "I can have problems but I now know how to manage myself and am able to find a solution." Another participant indicated that, when working with his peers, he is often asked how he knows so much about how to help others; another participant attributed his knowledge of helping others to the skills he gained in the HST program. One participant stated that the HST trainings allowed him to "know how to help the other Haitian survivors to treat themselves," effectively fostering sustainability and self-sufficiency in Haiti. Participants further reported an appreciation of the increased social support and networking opportunities they experienced directly at the trainings and also through working as local HST volunteers. All four participants noted that working with others during this challenging time was a source of comfort and strength.
The HST volunteers also experienced some difficulties. They frequently witnessed the suffering of others when volunteering. One participant had been a university student studying psychology before the earthquake, but felt that listening to people's stories of grief and loss required him to engage with others in a manner that exceeded his college training: "I found myself working with traumatized people, and that was one of the most difficult things for me-to work with people that were coping with a lot of bad stuff in life." In addition to the potential psychological distress involved with volunteering, participants noted difficulties related to logistics surrounding HST volunteering. During the HST trainings in which the Haitian volunteers learned the HST curriculum, there were difficulties with translation from English to French-Creole that the volunteers anticipated would negatively impact the Haitians' ability to learn the material. The participants also mentioned frustration with a lack of physical resources or materials (for example, water, medical supplies) to provide for those in need.

Following their training in the HST program, participants reported experiencing a greater degree of futureoriented thinking, such as finding fulfillment in life and having a renewed sense of hope. A participant reported that through his experience with HST he was able to learn how to calm and comfort others, which has provided him with a great sense of fulfillment. Similarly, another participant considered the HST skills to be a tool that local people may use to help rebuild Haiti and emphasized that "there is still hope because those relief workers are already gone, so you, yourself, who is staying, you need to fight to get another change in the country and to get it working."

\section{Discussion}

Prior research has connected many psychological benefits with those who volunteer ( $\mathrm{Li}$ and Ferraro 2005; Hildago et al. 2013), but information about the potential benefits of volunteerism in survivor populations is limited. Drawing on past research, we anticipated that the unique context of survivor populations (lack of resources, altered social connections, loss of relationships) would increase the likelihood that they would benefit from the act of volunteering.

In our study, positive evidence emerged supporting the psychological benefits experienced by volunteers in the post-disaster milieu. Through taking an active role in the recovery process in their country, Haitian volunteers were able to connect meaningfully to others, and experience a renewed sense of hope and purpose as a result of being a volunteer with the HST.

Part of the success of the HST program seems to be due to the ongoing, immediate needs that are present in 
survivor populations. All of our locally trained Haitian volunteers were themselves survivors who experienced considerable loss following the earthquake-loss of housing, loss of income, loss of family members, and loss of resources. The Haitian HST volunteers valued the training curriculum because it gave them a resource in the form of knowledge and support that they could share with members of their community. The Haitian HST volunteers were able to offer something to those around them who were hurting and contribute to the process of healing. While self-efficacy was not specifically measured through our analysis, the combination of a sense of purpose, pleasure in having something to offer others, and a genuine hope that they as volunteers can be agents of positive change seems to reflect the core tenets of the concept of self-efficacy (Bandura and Schunk 1981). Because of this implied presence of increased self-efficacy within our interviewed participants, we believe it is reasonable to anticipate that their resiliency would also be positively impacted by their involvement as volunteers.

Furthermore, because the Haitian HST volunteers are also embedded members of their communities, the knowledge and skills they acquired through the HST program are accessible to their communities not only in the purposeful transmission of support to others, but also in the natural transactions of everyday life of the volunteers. This natural transmission of support is especially important when considering the nature of a sustainable practice. Unlike expatriate volunteers who arrive quickly, dispense aid for a limited time and then leave, the Haitian HST volunteers are able to offer aid that is readily available at all times and in an ongoing way because they are members of their communities. While the data indicate that volunteers experience a sense of personal self-efficacy in the acquisition and dispensation of knowledge and skills, the communities as a whole also benefit, which contributes to healthy community capacity building.

Despite efforts to obtain interviews that would be informative regarding the effectiveness of the HST curriculum, there were limitations to our study that require examination. First, all of our volunteers spoke FrenchCreole and interviews were obtained through translators. We diligently interviewed and hired skilled translators; however, due to the sensitive and personalized nature of narrative inquiry analysis, it is possible that some nuances of the narrative stories of our interviewees manifested as less informative because of the language barrier. Additionally, our sample was small and homogenous. The HST curriculum is a relatively new program, rooted in evidencebased disaster mental health practices, but with limited experiential support. Although nearly 400 Haitian volunteers were trained through the HST program by other local Haitian trained trainers in the 2 years following the 2010 earthquake, the number of Haitian HST volunteers who had played an active enough role (that is, attended at least two trainings; worked 6 months as HST volunteer) to be considered for participation in our study was significantly limited. Another recruitment difficulty was communication with potential participants in post-disaster Haiti. Frequently changing housing locations and phone numbers were among the most common problems.

Furthermore, the culture in Haiti is traditionally maledominated. Men typically pursue activities and positions outside the home. Following the earthquake, the unemployed population was largely comprised of men, while women continued to carry out their traditional roles of caring for the family's immediate needs of food and shelter. Men, relative to women, reported more availability to volunteer because they were unable to work and were not tasked with managing the concerns of their households. Because of this gender disparity, we had far more men than women who were able or willing to volunteer their time through the HST. Of the approximately 500 local Haitian volunteers trained through the HST program, $<10$ individuals were women.

While the influence of specific, cultural factors within Haiti, and the small sample size within our study limit our ability to generalize our findings too broadly, this is the preliminary testing of a curriculum that is gaining momentum within the field of disaster response and will continue to be tested across cultural groups with more diverse samples. We anticipate that we will continue to find positive increases in psychological well-being and resilience, both for the volunteers and their survivor communities.

\section{Conclusion}

Despite the limitations of the current study, there are significant positive implications for the results found here. It is clear that volunteerism in general is of psychological benefit to those who are supported, as well as to the volunteers themselves. In the context of a large-scale disaster recovery situation, the benefits of training local survivors as volunteers within the affected communities are supported conceptually by previous research, and substantively by the self-reporting of our interviewed participants. The Haitian HST volunteers in this study consistently reported an increase in personal resilience, interpersonal empowerment, and social connection. It is recommended that further research be undertaken with additional volunteers and in varying international communities. Additional trainings with the HST curriculum are currently under way with Syrian refugees in Jordan, and future research will examine whether similar positive effects emerge for those 
trained in the context of a large-scale man-made disaster (for example, civil war). Resilience in the context of disaster recovery is an essential component of psychological health. By identifying concrete methods through which resilience can be increased among surviving populations, international disaster work will become more effective and sustainable.

Open Access This article is distributed under the terms of the Creative Commons Attribution License which permits any use, distribution, and reproduction in any medium, provided the original author(s) and the source are credited.

\section{References}

ARC (American Red Cross). 2009. Our history: A brief history of the American Red Cross. http://www.redcross.org/about-us/history. Accessed 24 April 2014.

Bandura, A., and D.H. Schunk. 1981. Cultivating competence, selfefficacy, and intrinsic interest through proximal self-motivation. Journal of Personality and Social Psychology 41(3): 586-598.

Brown, K.M., R. Hoye, and M. Nicholson. 2012. Self-esteem, selfefficacy, and social connectedness as mediators of the relationship between volunteering and well-being. Journal of Social Service Research 38(4): 468-483.

Charney, D.S. 2004. Psychobiological mechanisms of resilience and vulnerability: Implications for successful adaptation to extreme stress. American Journal of Psychiatry 161(2): 195-216.

Creswell, J.W., W.E. Hanson, V.L. Plano, and A. Morales. 2007. Qualitative research designs: Selection and implementation. The Counseling Psychologist 35(2): 236-264.

Gelkopf, M., R. Berger, P. Ryan, and S.J. Cotton. 2008. The impact of "Training the Trainers" course for helping tsunami-survivor children on Sri Lankan disaster volunteer workers. International Journal of Stress Management 15(2): 117-135.

Gilgun, J.F. 2009. Qualitative research and family psychology. In The Wily-Blackwell handbook of family psychology, ed. J.H. Bray, and M. Stanton, 85-99. Malden, MA: Wiley-Blackwell.

Glister, M.E. 2012. Comparing neighborhood-focused activism and volunteerism: Psychological well-being and social connectedness. Journal of Community Psychology 40(7): 769-784.

Haverkamp, B.E., and R.A. Young. 2007. Paradigms, purpose, and the role of the literature: Formulating a rationale for qualitative investigations. The Counseling Psychologist 35(2): 265-294.
Hildago, C.M., P. Moreno-Jiménez, and J. Quiñonero. 2013. Positive effects of voluntary activity in older adults. Journal of Coтmunity Psychology 41(2): 188-199.

Jacobs, G. 2007. The development and maturation of humanitarian psychology. American Psychologist 62(8): 932-941.

King, D., and C. MacGregor. 2000. Using social indicators to measure community vulnerability to natural hazards. Australian Journal of Emergency Management 15(3): 52-57.

Li, Y., and K.F. Ferraro. 2005. Volunteering and depression in later life: Social benefits or selection process? Journal of Health and Social Behavior 46(3): 68-84.

Lieblich, A., R.T. Tuval-Mashiach, and T. Zilber. 1998. Narrative research: Reading, analysis, and interpretation. Thousand Oaks, CA: Sage.

Mitchell, J.T., and G.S. Everly. 2001. The basic critical incident stress management course: Basic group crisis intervention. Ellicott City, MD: International Critical Incident Stress Foundation.

Ponterotto, J.G. 2005. Qualitative research in counseling psychology: A primer on research paradigms and philosophy of science. Journal of Counseling Psychology 52(2): 126-136.

Poulin, M.J. 2014. Volunteering predicts health among those who value others: Two national studies. Health Psychology 33(2): $120-129$.

Reissman, C.K. 1993. Narrative analysis. Qualitative research methods series 30. Newbury Park, CA: Sage.

Richie, J., J. Lewis, and G. Elam. 2003. Designing and selecting samples. In Qualitative research practice. A guide for social science students and researchers, ed. J. Ritchie, and J. Lewis, 77-108. Thousand Oaks, CA: Sage.

Ruzek, J.I., M.J. Brymer, A.K. Jacobs, C.M. Layne, E.M. Vernberg, and P.J. Watson. 2007. Psychological first aid. Journal of Mental Health Counseling 29(1): 17-49.

Schreiber, M. 2010. The PsySTART disaster mental health triage and incident management system. http://www.cdms.uci.edu/pdf/psy start-cdms02142012.pdf. Accessed 15 May 2014.

Shelly, L. 2011. A qualitative analysis of the experiences of critical incident stress debriefers. Ph.D. dissertation, Seattle Pacific University.

Tang, F., E. Choi, and N. Morrow-Howell. 2010. Organizational support and volunteering benefits for older adults. The Gerontologist 50(5): 603-612.

Thoburn, J.W., K. Mauseth, S. Cecchet, T. MacGuire, and K. Adams. 2014. Health support team: A qualitative analysis of the outcomes from a mental health training program for community-volunteers in Haiti. International Perspectives in Psychology: Research, Practice, Consultation. 